\title{
¿Es divertido aprender con Kahoot!?: la percepción de los estudiantes
}

\section{Carmen Ruiza $^{\text {a }}$, Rocío Martínez ${ }^{a}$, Elia García, Cristina Pedrosa ${ }^{a}$ y Ana Licerán ${ }^{b}$}

${ }^{a}$ Departamento de Organización de Empresas, Marketing y Sociología, Universidad de Jaén. Paraje Las Lagunillas, s/n. 23.071- Jaén. E-mail: cruiz@ujaen.es, rmartine@ujaen.es, cpedrosa@ujaen.es, egarcia@ujaen.es

${ }^{\mathrm{b}}$ Departamento de Economía Financiera y Contabilidad, Universidad de Jaén. Paraje las Lagunillas, s/n. 23.071- Jaén- E-mail: aliceran@ujaen.es

\begin{abstract}
The introduction of gamification in the university classroom, through new technologies is a powerful tool that is giving great results. The main objective at this work is to know and analyze the assessment that students make of the use of the Kahoot! as a tool for evaluating your learning process. In addition, we wanted to check if there are significant differences in said assessment according to degree, age and sex. The results come to show very positive results. Students value the tool as very interesting and fun, while allowing them to take a more active role in class, get more involved, improve their study and understanding of content and, ultimately, have a greater motivation for their learning. Further we have verified that there has been a significant improvement in class attendance throughout the semester.
\end{abstract}

Keywords: Active methodologies, gamification, Kahoot!, m-learning, assessment, evaluation, teaching-learning process, motivation, participation.

\section{Resumen}

La introducción de la gamificación en el aula universitaria, de la mano de las nuevas tecnologías es una potente herramienta que está dando magnificos resultados. En este trabajo nuestro principal objetivo ha sido conocer y analizar la valoración que los estudiantes hacen del uso de la aplicación Kahoot! como herramienta de evaluación de su proceso de aprendizaje. Además, hemos querido comprobar si existen diferencias significativas de dicha valoración según titulación, edad y sexo. Los resultados vienen a mostrar resultados muy positivos. Los alumnos valoran la herramienta como muy interesante y divertida, a la vez que les ha permitido adoptar un rol más activo en clase, implicarse más, mejorar su estudio y comprensión de contenidos y, en definitiva, tener una mayor motivación de cara a su aprendizaje. Añadido a lo anterior, hemos comprobado que ha habido una mejora importante de la asistencia a clase durante todo el cuatrimestre.

Palabras clave: metodologías activas, gamificación, kahoot!, m-learning, valoración, evaluación, proceso enseñanza-aprendizaje, motivación, participación. 


\section{Introducción}

La nueva concepción de la enseñanza, en todos sus niveles, y especialmente en el nivel universitario, apunta hacia un aprendizaje más activo, centrado en el estudiante, que incluya vías de aprendizaje flexibles y que sea capaz de reconocer las competencias obtenidas por el alumnado tanto dentro como fuera de los planes de estudio oficiales. Con esta nueva concepción metodológica, el centro del proceso debe ser el estudiante y su aprendizaje, por lo que el profesor tiene que regular su actuación al servicio del aprendizaje efectivo del mismo (Calderón y González, 2009). A la vez, también se demanda un cambio de rol por parte de los estudiantes, de manera que estos pasen de ser sujetos pasivos y se conviertan en los autores que construyen el conocimiento que deben adquirir.

En este contexto, el "informe Horizon 2017" (Adams et al, 2017) recoge entre las tendencias y recomendaciones a corto plazo, además del diseño de aprendizajes mixtos y colaborativos, la introducción del aprendizaje móvil o m-learning. Esta tendencia metodológica es capaz de estimular la participación activa del alumnado a través de distintos dispositivos como las tablets, smartphones, portátiles..., convirtiéndose éstos en potentes herramientas educativas que pueden proporcionar múltiples ventajas (Fuertes, 2016); así como facilitar el aprendizaje y adquisición de habilidades de manera autónoma, en cualquier momento y lugar, gracias al uso de dichos dispositivos móviles (Brazuelo y Gallego, 2011).

Junto a esta tendencia, cada vez son más las experiencias de introducción de la gamificación o ludificación en el aula. Aunque existen diferentes definiciones, la gamificación consiste en la utilización de las mecánicas del juego, su estética y sus estrategias para involucrar a la gente, motivar a la acción, promover el aprendizaje y resolver problemas (Kapp, 2012). La importancia de la participación del alumno en su propio aprendizaje o el feedback inmediato que recibe, son algunas de las razones que justifican la introducción de la gamificación en el aula. El proceso es muy simple, el alumno aprende jugando, con lo que alcanza una mayor satisfacción en las actividades educativas, lo que aumenta la retención de lo aprendido y le estimula para continuar haciéndolo (Monedero y Castro, 2018). Numerosos trabajos (p. ej. Del Cerro, 2015; Villalustre y Moral, 2015; Wang y Lieberoth, 2016; Barnes, 2017; Chaiyo y Nokham, 2017; García et al., 2017; Llerena y Rodriguez, 2017; Peña et al.,2017; Rodriguez, 2017; Roger et al., 2017 y San Miguel et al., 2017) muestran los resultados positivos de la gamificación en el ámbito docente, mejorando notablemente la atención de los estudiantes y haciendo más entretenido el proceso de aprendizaje (Sainz et al., 2018).

Por tanto, el tándem gamificación-nuevas tecnologías parece que ofrece al profesorado nuevas posibilidades de enseñanza-aprendizaje que, en muchos casos, ya están siendo reconocidas como fuente de innovación.

En este contexto, si bien existe un amplio abanico de aplicaciones (Socrative, Quizizz, Googleforms, Brainscape, Cerebriti, etc.) que favorecen la transición hacia metodologías de aprendizaje más activas e innovadoras, Kahoot! reúne características que la hacen propicia para ser utilizada como herramienta de aprendizaje y evaluación (Wang y Lieberoth, 2016). Kahoot! es una plataforma on line desarrollada en 2013 por el profesor Alf Inge Wang, de la Norwegian University Of Science And Technology. Es una herramienta que conjuga el uso 
de nuevas tecnologías con la gamificación. Permite crear encuestas, cuestionarios y discusiones, obteniendo feedback de los alumnos en tiempo real.

El uso de Kahoot! en diferentes ámbitos educativos ha sido estudiado previamente, ofreciendo buenos resultados (Mu y Paparas, 2015; Grinias, 2017; Iwamoto et al., 2017; Rodriguez, 2017; Alcover et al., 2018; Sempere, 2018; Sainz et al., 2018). Las investigaciones concluyen que Kahoot! es una buena herramienta para realizar actividades en el aula, que contribuye a mejorar la participación de los estudiantes, quienes lo perciben como un juego y gracias a lo cual, se fomenta una relación positiva entre el grupo y aumenta la tasa de asistencia a clase.

\section{Objetivos}

Hasta el momento son muy pocos los trabajos que abordan el uso de esta aplicación en el área de las ciencias sociales. Por esta razón y a la vista de los resultados de los trabajos citados anteriormente y de los beneficios de la gamificación en el proceso de enseñanza aprendizaje, el objetivo que se persigue con este trabajo es conocer y analizar la valoración que los estudiantes hacen del uso de la aplicación Kahoot! como herramienta de evaluación de su proceso de aprendizaje. Más concretamente nos planteamos los siguientes objetivos específicos: introducir la gamificación como instrumento de trabajo en el aula a través del mlearning; conocer la valoración de los estudiantes sobre dicha herramienta; identificar si existen diferencias significativas de dicha valoración según titulación, edad y sexo; y conocer los aspectos positivos y negativos que los estudiantes destacan de esta dinámica metodológica.

\section{Desarrollo de la innovación}

Tanto el desarrollo de esta experiencia de gamificación en el aula universitaria como la difusión de sus resultados, ha sido posible gracias a la concesión del proyecto de Innovación Docente titulado "Una experiencia de gamificación en el aula: evaluamos con Kahoot!", concedido por la Universidad de Jaén en el marco del "Plan de Innovación Docente e Incentivación de las buenas prácticas docentes” (con código PID32_201819).

La evaluación con la aplicación Kahoot! ha sido desarrollada en cuatro asignaturas de tres grados universitarios (Grado en Administración y Dirección de Empresas, Grado en Finanzas y Contabilidad, Grado en Relaciones Laborales y Recursos Humanos) y en el Master en Administración de Empresas, de la Facultad de Ciencias Sociales y Jurídicas, de la Universidad de Jaén.

Se ha trabajado con grupos relativamente reducidos y esto ha facilitado mucho la puesta en práctica de esta herramienta. Concretamente, los estudiantes que han trabajado en el total de estas cuatro asignaturas han sido 133. Mostramos a continuación cómo ha sido su distribución: 
- 35 alumnos de Organización y Administración de Empresas, de primer curso del Grado en Relaciones Laborales y Recursos Humanos (RRLL y RRHH).

- 43 alumnos de Dirección Estratégica II, de cuarto curso del Grado en Administración y Dirección de Empresas (ADE).

- 28 alumnos de Gestión de Recursos Humanos, de segundo curso del Grado en Finanzas y Contabilidad (FYCO).

- 27 alumnos de Dirección de Recursos Humanos del Master en Administración de Empresas (MBA).

En las cuatro asignaturas se ha seguido el mismo procedimiento. Al finalizar cada uno de los temas, se ha preparado una prueba Kahoot!, con el objetivo de comprobar el nivel de conocimiento asimilado por los estudiantes sobre dicha materia. Todas las preguntas han sido elaboradas por las profesoras, estando relacionadas con los contenidos abordados en cada unidad. Según el orden de dificultad, tanto de los enunciados de las preguntas, como de las respuestas, se ha estimado un tiempo prudencial de respuesta, que ha oscilado entre los 2060 segundos.

A medida que se iban aplicando las preguntas y una vez finalizado el tiempo de respuesta y comprobados los errores que hubiera, se ha ido realizando feedback (profesora-alumnos o alumno-alumno), dedicando un tiempo a resolver posibles dudas sobre las respuestas elegidas.

Para conocer la valoración de los alumnos sobre la utilidad de Kahoot! como herramienta de evaluación, más allá de nuestras propias valoraciones, los días próximos a finalizar el primer cuatrimestre, se les pidió que completaran un cuestionario. Este cuestionario ha sido voluntario y anónimo. En él, se les pedía que valoraran su experiencia con el uso de la aplicación Kahoot!, usando una escala Likert que iba desde 1 (muy en desacuerdo) hasta el 5 (muy de acuerdo). Se incluían preguntas acerca sobre motivación, interés, diversión, participación, etc. Además, se le plantearon dos preguntas abiertas para que los estudiantes valoraran los aspectos positivos (lo que más te ha gustado) y los negativos (lo que menos te ha gustado), de esta experiencia.

El cuestionario ha sido respondido por 111 estudiantes, lo cual indica una tasa de respuesta del $82 \%$. El reparto de la muestra entre asignaturas y titulaciones es más o menos equitativo ( 25 cuestionarios en FICO y en ADE; 27 en MBA y 34 en RRLL) y aparece reflejado en porcentajes en la figura 1. 


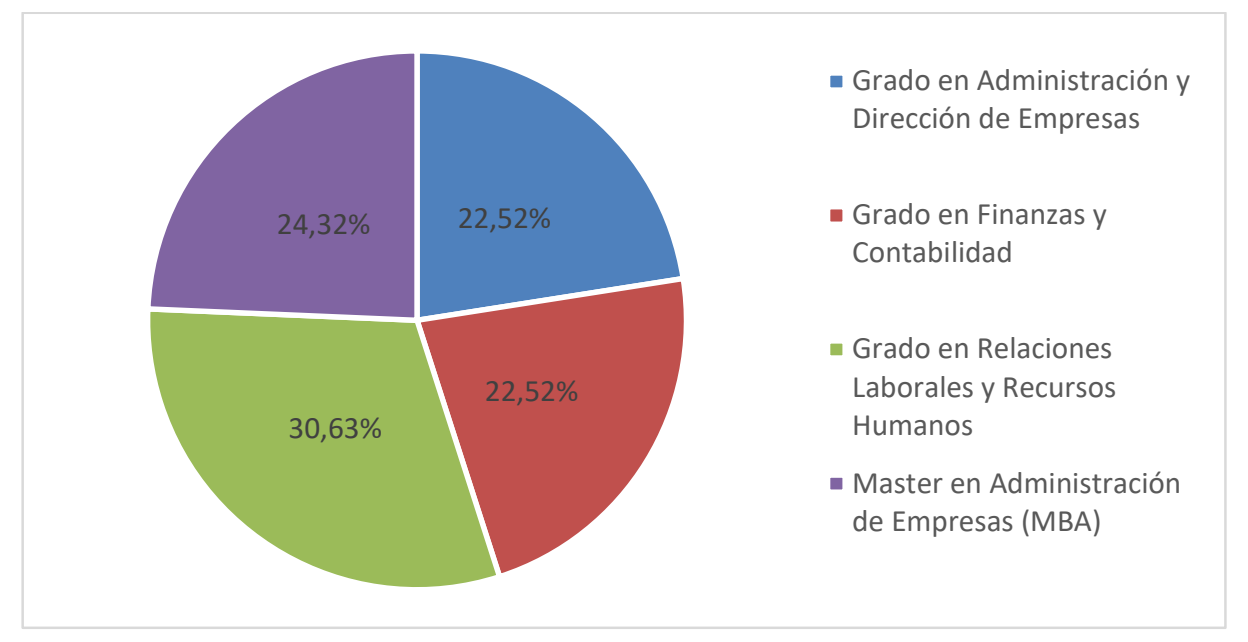

Fig. 1.Distribución de estudiantes que han respondido al cuestionario por titulación.

Por otra parte, nos parece adecuado comentar cómo se ha distribuido dicha muestra en cuanto a sexo, también bastante equitativo (ver figura 2) y grupos de edad (ver figura 3). En este sentido, los estudiantes, como era de esperar, se encuentran mayoritariamente por encima de los 18 años y por debajo de los 22-23 años. Este rango de edad se corresponde con los cursos en los que se encuentran las asignaturas elegidas para el estudio. En concreto, la asignatura de Dirección Estratégica II es de $4^{\circ}$ curso del Grado en ADE; la asignatura de Gestión de Recursos Humanos se encuentra en $2^{\circ}$ curso del Grado en FICO; y la asignatura de Organización y Administración de Empresas se encuentra en el $1^{\circ}$ curso del Grado en RRLL. No obstante, al haber incluido también una asignatura de master en nuestro estudio, tenemos algunos estudiantes que sobrepasan los 30 años, siendo la edad máxima 39 años.

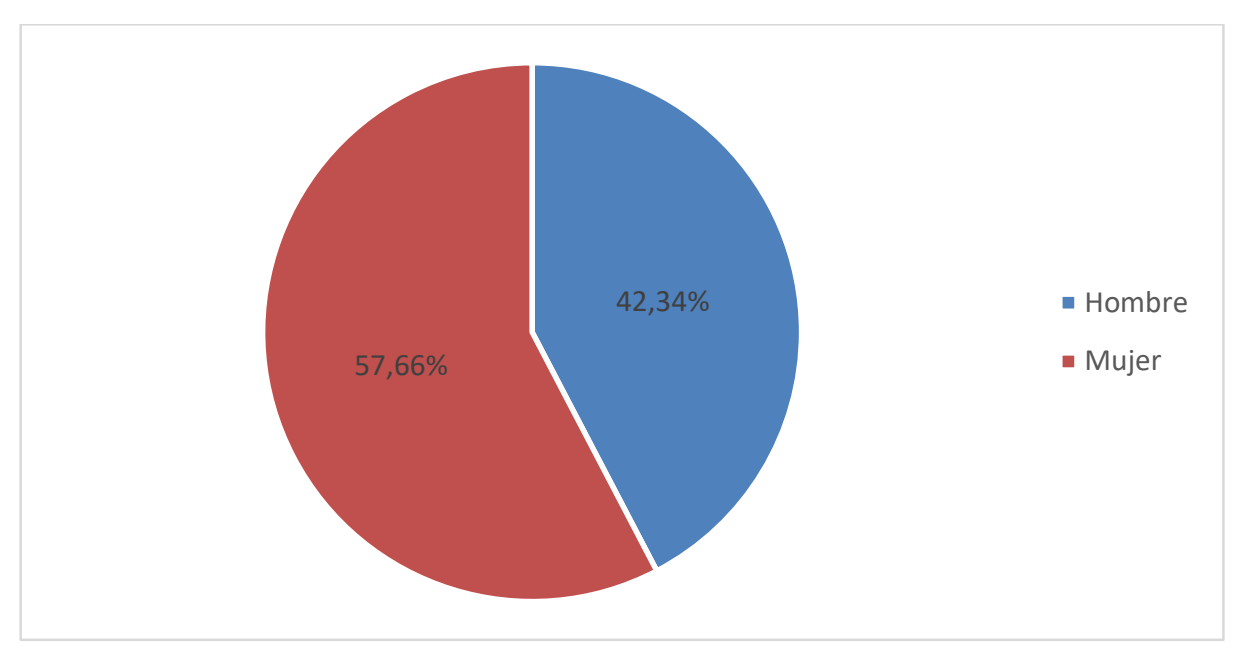

Fig.2. Distribución de estudiantes por sexo. 


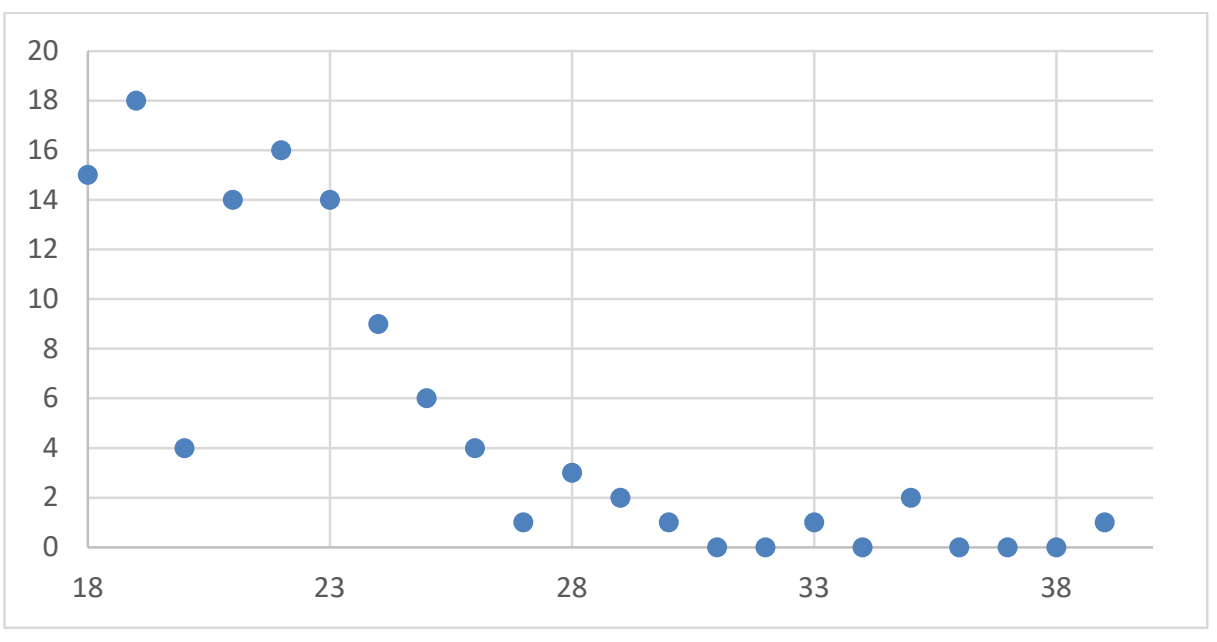

Fig.3. Distribución de estudiantes por edad.

\section{Resultados}

En relación al segundo de nuestros objetivos (conocer la valoración de los alumnos de la utilización de Kahoot! como herramienta de evaluación sobre el proceso de aprendizaje), la primera cuestión que planteamos a nuestros estudiantes era en qué medida el uso de esta herramienta en clase les había parecido interesante. En este caso, la valoración media de todos los estudiantes ha sido de un 4.64 con una desviación típica de 0.569 . Por titulaciones, tal y como podemos observar en la figura 4, los estudiantes de FICO son los que han considerado en mayor medida que esta herramienta es de interés, seguidos por los estudiantes del MBA a quienes quizás es a quien más ha sorprendido el uso de esta nueva metodología. En el extremo opuesto nos encontramos a los estudiantes de RRLL, si bien la valoración es superior en todo momento a 4 , un valor más que satisfactorio.

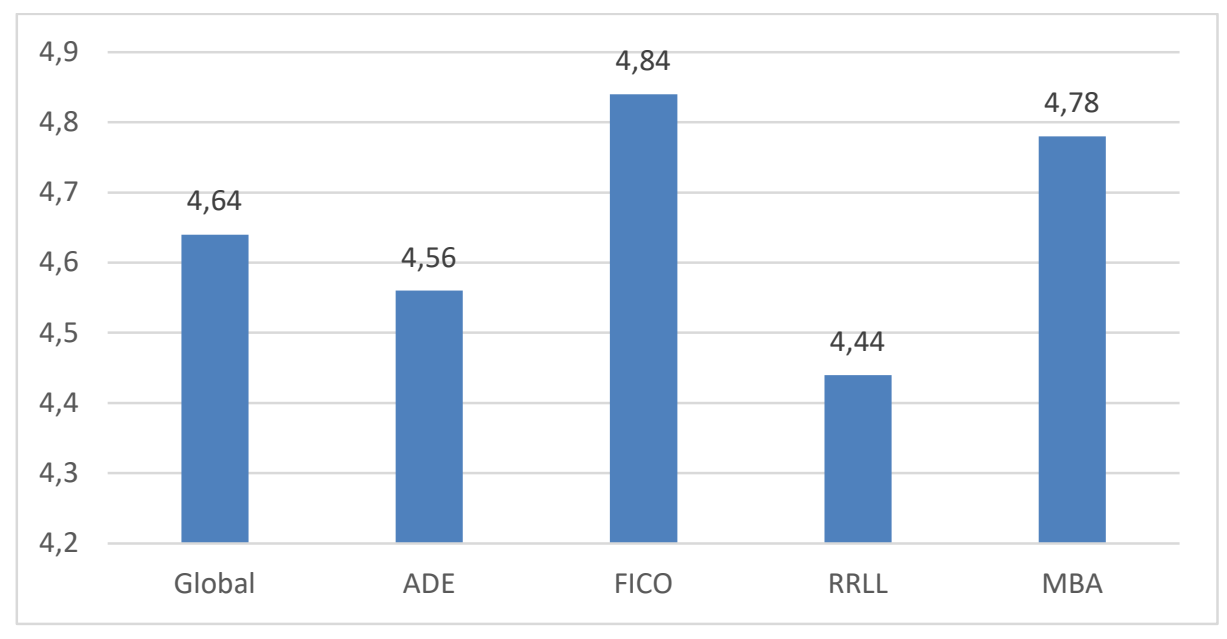

Fig.4.la herramienta Kahoot! me ha resultado interesante. 
En cuanto a la segunda pregunta, les planteábamos a nuestros estudiantes si el uso de Kahoot! había mejorado su motivación en clase. En este caso la valoración media también ha superado el 4, aunque con un valor un poco más bajo que el anterior (4.32) y una mayor desviación típica (0.863). Igual que en el caso anterior, y como se puede observar en la figura 5, los estudiantes de FICO son los que han dado una mayor valoración a este ítem y los de RRLL los que menor puntuación han concedido.

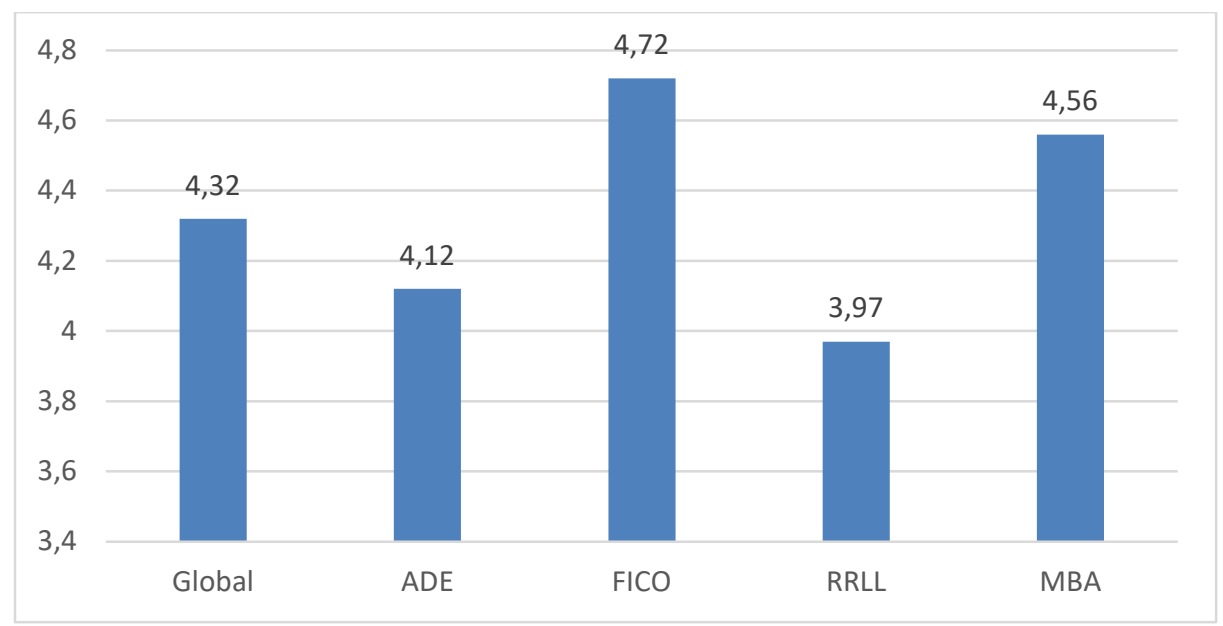

Fig.5.La herramienta Kahoot! ha mejorado mi motivación en clase.

Otro elemento que hemos valorado ha sido en qué medida se han divertido usando la aplicación Kahoot!. En este caso, la valoración también es muy positiva, con una media de 4.59 (desviación típica de 0.680 ). Realmente, las profesoras ya habíamos constatado esto en las clases puesto que lo cierto es que era un momento bastante alegre y entretenido para ellos, a pesar de tratarse de una evaluación (curiosamente). Por titulaciones, tal y como presentamos en la figura 6, vuelven a repetirse los patrones que hemos comprobado hasta ahora, con FICO con la mayor valoración, seguida del MBA y RRLL con la valoración más baja.

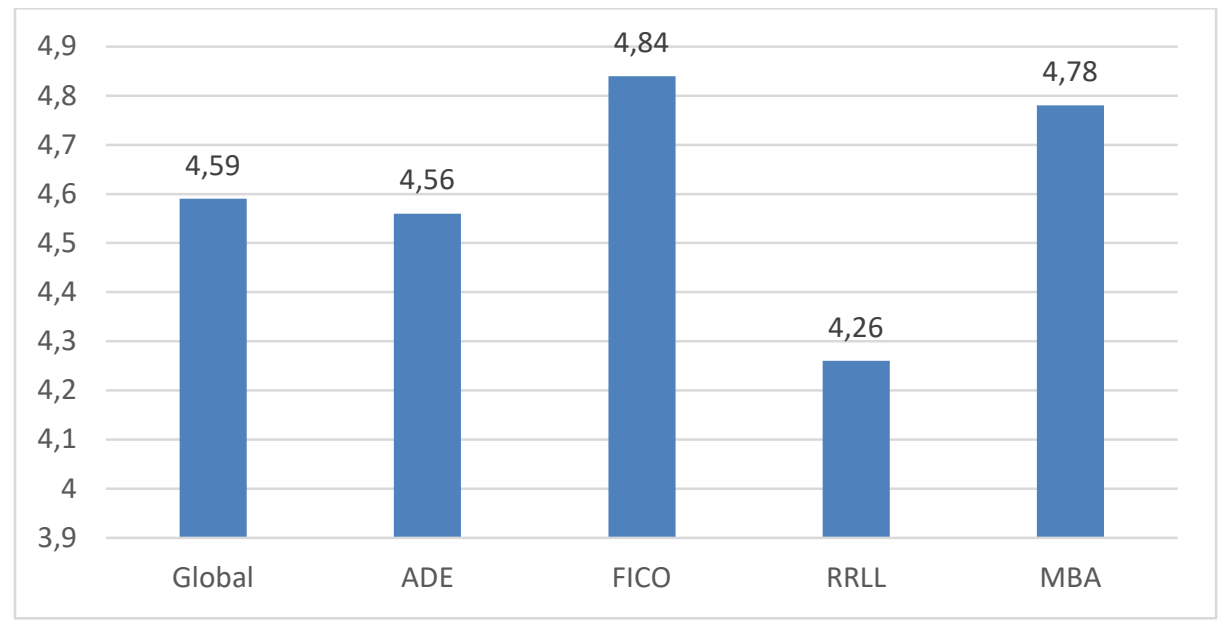

Fig.6. Me he divertido con Kahoot! 
La competitividad que se genera entre los compañeros de clase a la hora de realizar estos test era palpable en clase, pues entre ellos "se picaban" para ver quién estaba en el pódium y quién ganaba cada uno de los test. Por este motivo, quisimos valorar también en qué medida consideran que esa competitividad es positiva. En este caso, la valoración media también es muy alta (4.45, con una desviación típica de 0.737$)$. Por titulaciones, comprobamos en la figura 7 que esta vez son los estudiantes del MBA los que otorgan una mayor puntuación a este ítem y son los de ADE los que puntúan más abajo.

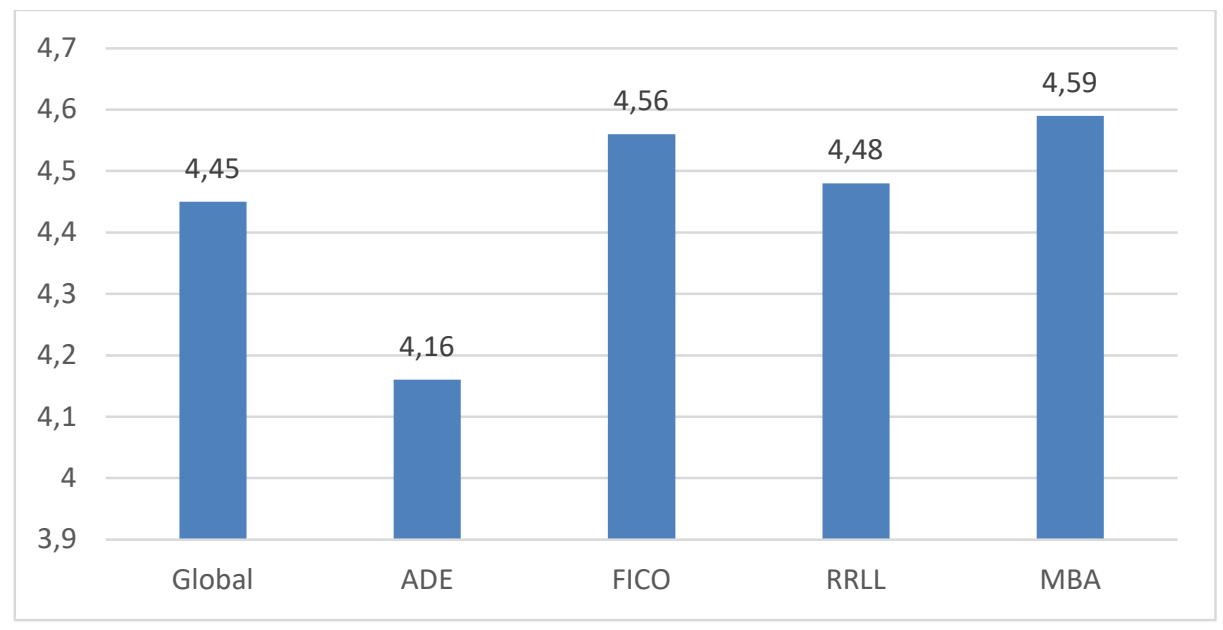

Fig. 7.La competitividad entre compañeros al usar Kahoot|! es positiva.

También quisimos saber en qué medida el uso de Kahoot! había ayudado a nuestros estudiantes a participar más activamente en clase. Este ítem puntúa un poco más bajo que los anteriores (4.26, con desviación típica de 0.891). Por titulaciones, nuevamente son FICO y MBA los que puntúan más elevado y $\mathrm{ADE}$ que baja por debajo de 4 se queda como el grupo de estudiantes que menos han valorado este ítem (ver figura 8).

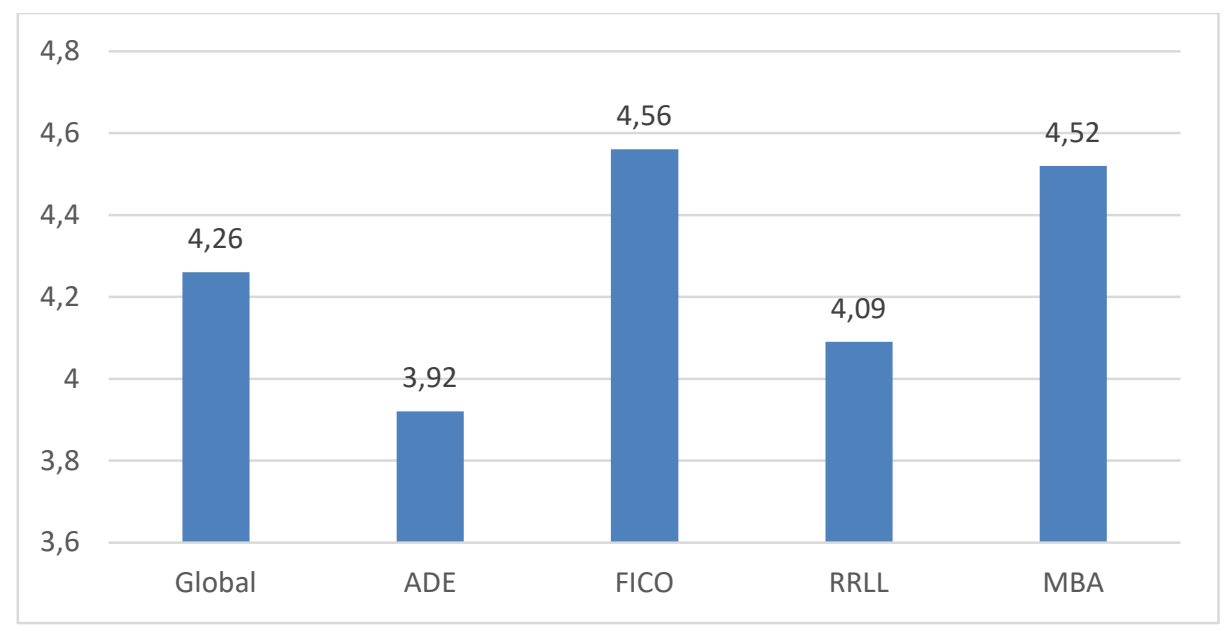

Fig.8.Kahoot|! me ha hecho participar más activamente. 
Del mismo modo, creemos que el hecho de utilizar estos test con Kahoot! para cada unidad o tema, es útil para los estudiantes al "obligarles" a estudiar y preparar la asignatura día a día. También consideramos útil esta herramienta para aclarar algunos conceptos ya explicados, que no se han llegado a entender completamente por parte del alumnado. En los dos casos, los estudiantes han opinado igual que las profesoras, otorgándole a ambos elementos una valoración global superior al 4 (4.13 y 4.41, con desviaciones típicas de 0.964 y 0.779 , respectivamente). Por titulaciones, tal y como podemos observar en la figura 9, los estudiantes de FICO y MBA son los que vuelven a puntuar más alto en estas cuestiones. Especialmente relevante es la valoración en el master de la segunda cuestión (con un 4.74 es la más alta de todas). En el extremo opuesto, los estudiantes de ADE son quienes vuelven a puntuar más bajo ambas cuestiones.

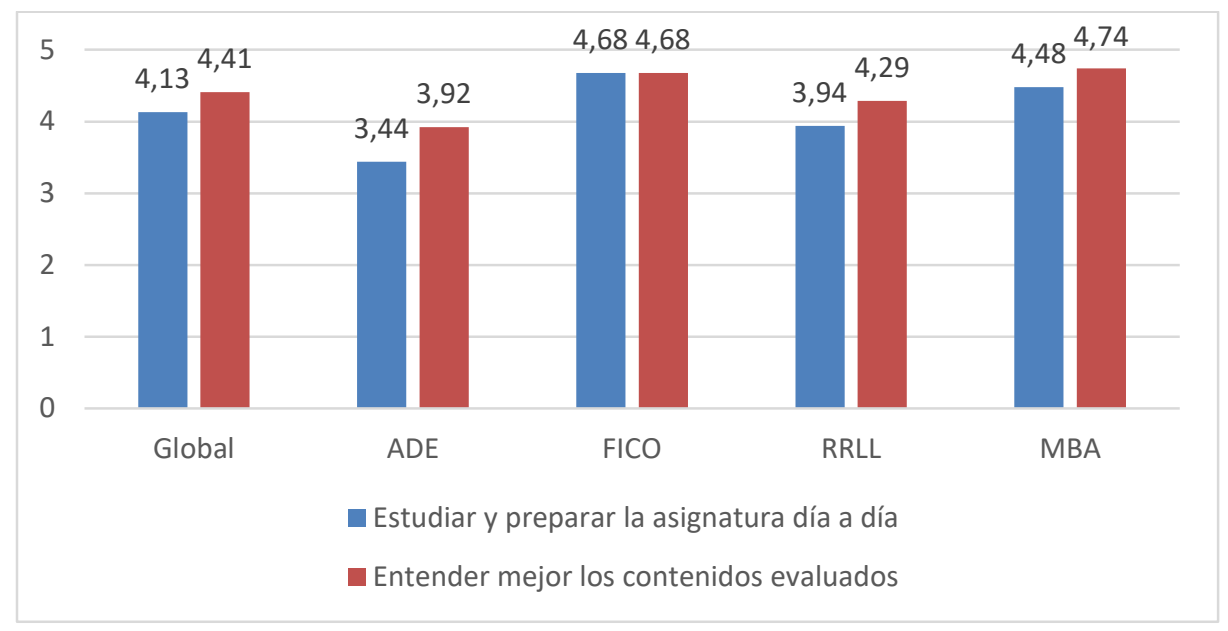

Fig.9. Kahoot! me ha servido para estudiar y preparar la asignatura día a día y para entender mejor los contenidos evaluados

Por último, quisimos analizar en qué medida los estudiantes ven positivo el hecho de que la puntuación obtenida en los Kahoot! sea tenida en cuenta como una parte de la valoración final de la asignatura; esto es, realizar una evaluación sumativa donde los tests de Kahoot! puntúen en el apartado de Asistencia y participación en clase (aunque en realidad no suponía ni siquiera el 10\% de la calificación). En realidad, esto sucedió así en las asignaturas de FICO y RRLL. En el MBA se realizaron como valoración de la parte teórica de la asignatura (por tanto, fue donde más se tuvo en cuenta) y en el grado en ADE no se les tuvo en cuenta en la calificación final, sirviendo únicamente a la profesora como valoración del aprendizaje individual. A pesar de este diferente uso, todos los estudiantes consideran esta idea como muy buena, puesto que la valoración media obtenida en esta pregunta es de 4.39 (con una desviación de 0.822). Por titulaciones, como se observa en la figura 10, en todos los casos se supera la valoración de 4, siendo en el MBA y en FICO nuevamente donde se alcanza una mayor valoración. 


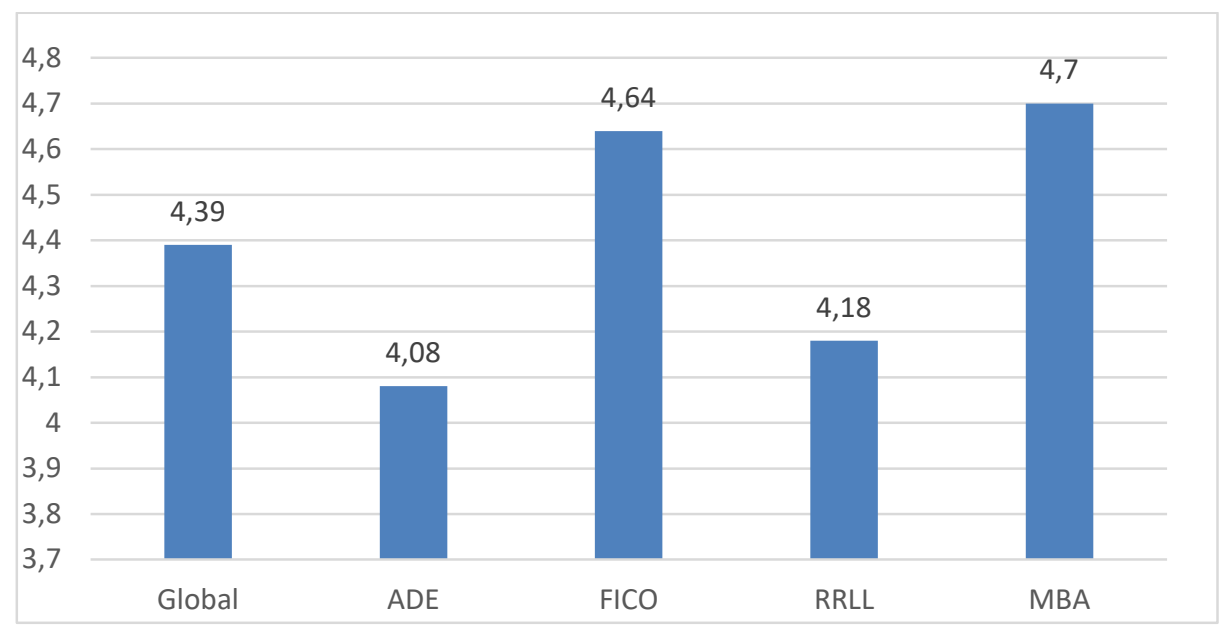

Fig.10. Me parece adecuado usar los resultados de Kahoot! como parte de la valoración de mi participación en la asignatura

En relación al tercero de nuestros objetivos, quisimos analizar si existen diferencias significativas entre los grupos de titulaciones mediante un estudio de tablas de contingencia (con Chi-cuadrado) y un estudio ANOVA. En la tabla 1 podemos comprobar como las diferencias sí son estadísticamente significativas en varios de los ítems analizados, con una significación del 95\% (*) y hasta del 99\% (**) en algunos casos.

Tabla 1: ANOVA y Chi-cuadrado por Titulación

\begin{tabular}{lcc}
\hline & $\begin{array}{c}\text { ANOVA } \\
\text { p-value }\end{array}$ & $\begin{array}{c}\text { Chi-cuadrado de Pearson } \\
\text { Sig, asintótica }\end{array}$ \\
\hline Interesante & $0.023^{*}$ & $0.014^{*}$ \\
Motivación & $0.002^{* *}$ & $0.020^{*}$ \\
Diversión & $0.003^{* *}$ & $0.003^{* *}$ \\
Competitividad & 0.138 & 0.113 \\
Participación & $0.017^{*}$ & $0.021^{*}$ \\
Trabajo diario & $0.000^{* *}$ & $0.002^{* *}$ \\
Entender mejor & $0.000^{* *}$ & $0.002^{* *}$ \\
Adecuación & $0.006^{* *}$ & $0.006^{* *}$ \\
\hline
\end{tabular}

También hemos querido realizar este análisis pero considerando la edad como factor. Los resultados también muestran diferencias significativas en varios de los ítems (ver tabla 2).

Tabla 2: ANOVA y Chi-cuadrado por Edad

\begin{tabular}{cc}
\hline ANOVA & Chi-cuadrado de Pearson \\
p-value & Sig, asintótica \\
\hline
\end{tabular}




\begin{tabular}{lcc}
\hline Interesante & $0.000^{* *}$ & $0.000^{* *}$ \\
Motivación & $0.000^{* *}$ & $0.009^{* *}$ \\
Diversión & $0.000^{* *}$ & $0.000^{* *}$ \\
Competitividad & 0.214 & 0.124 \\
Participación & $0.000^{* *}$ & $0.000^{* *}$ \\
Trabajo diario & $0.050^{*}$ & 0.703 \\
Entender mejor & 0.099 & 0.344 \\
Adecuación & 0.147 & 0.054 \\
\hline
\end{tabular}

Finalmente, hemos de decir que por sexo no hemos encontrado ninguna diferencia significativa.

Por último, en relación al último de nuestros objetivos (conocer los aspectos positivos y negativos de esta dinámica metodológica), comentamos a continuación los resultados del análisis cualitativo realizado en las dos preguntas abiertas incluíadas en el cuestionario. Así, sobre lo positivo o lo que más ha gustado de la experiencia de Kahoot!, muchos de los estudiantes la definen como una herramienta fácil de usar, novedosa, dinámica y diferente. El hecho de utilizar esta aplicación es, para la mayoría, una actividad entretenida, amena y divertida, que genera un buen ambiente en clase entre los compañeros con una competitividad entre ellos sana y motivadora. La gran mayoría de los estudiantes considera también que ha sido útil para llevar la asignatura al día y entender mejor la materia (repiten dos de las preguntas que nosotros les dábamos, pero eso demuestra la importancia que ha tenido Kahoot! en este aspecto). Finalmente, varios de ellos también hacen referencia al aspecto positivo de recibir una retroalimentación inmediata (saber tus errores y por qué has fallado y tener tu calificación en el momento).

Sobre lo negativo, hay más unanimidad y se suelen repetir dos respuestas: los fallos que en ocasiones ha dado la aplicación y el poco tiempo que algunas veces tenían para responder. Este último aspecto es más indicado por las mujeres que por los hombres. La realidad del poco tiempo para responder, se corresponde con una necesidad de evitar la copia por parte del alumno. Los Kahoot!s antes de ser respondidos por los alumnos, son testados por el profesor responsable de la asignatura y el tiempo ofrecido para su resolución se considera suficiente (de 20 a 60 segundos según la pregunta). Hay que tener en cuenta también que las aulas son adecuadas para las clases, pero en el momento de la evaluación no existe espacio físico suficiente para separar a los alumnos y evitar así la "tentación de imitar al de al lado".

\section{Conclusiones}

Kahoot! se ha mostrado como una herramienta de evaluación muy positiva en el caso de las cuatro asignaturas en las que se ha utilizado. Más concretamente, podemos decir que ha sido una metodología de enseñanza-aprendizaje interesante, que ha hecho que los estudiantes 
adopten un rol mucho más activo, lo que ha provocado un aumento de su motivación e interés por la asignatura. Además, se ha mejorado la asistencia a clase, un tema que nos preocupaba especialmente desde hace varios años y que precisamente nos ha llevado a plantearnos introducir nuevas herramientas metodológicas en clase. En este sentido, pudimos comprobar que los estudiantes que comenzaron las clases asistiendo regularmente y realizando los primeros Kahoot!s se mantuvieron durante todo el cuatrimestre hasta el último día.

Además de estos aspectos, como principales fortalezas de la utilización del Kahoot! como herramienta de evaluación podemos concluir:

- Se ha mejorado el estudio y el aprendizaje de la asignatura. Las opiniones de los estudiantes corroboran que han llevado la asignatura al día (frente al habitual estudio varios días antes del examen) y que han comprendido mejor los principales conceptos vistos en el Kahoot!. Esto se debe al feedback, a que la herramienta permitía al profesorado detectar los fallos en la comprensión de los contenidos y volver a explicarlos, en algunas ocasiones, contando con los propios estudiantes.

- La realización de los Kahoot!s ha favorecido la participación de los alumnos. En ocasiones encontramos alumnos más introvertidos a los que les cuesta relacionarse con el resto y/o con el propio profesor. Estos alumnos han finalizado el cuatrimestre totalmente integrados en el grupo. El juego les ha servido como herramienta de enlace e integración en el aula.

- Los estudiantes valoran positivamente el hecho de que haya una evaluación sumativa en la asignatura que tenga en cuenta los resultados de los tests. De esta manera, ellos consideran que se valora el aprendizaje realizado a lo largo del cuatrimestre y la nota no depende sólo del examen final. Además, el conocer en el momento la calificación obtenida en el test les motivaba a seguir adelante sabiendo que ya habían acumulado algunos puntos para la nota final.

- Al utilizar esta herramienta se genera un clima positivo de competitividad entre ellos, siempre en un ambiente relajado, agradable y divertido que les hace "picarse" para ver si pueden desbancar al compañero que va en primera posición.

- Como han mostrado investigaciones previas, la herramienta de gamificación usada ha demostrado tener éxito por la interactividad que proporciona al alumnado que adopta un doble rol de jugador y aprendiz (Marín Suelves et al., 2018).

Como debilidades o principales aspectos que habría que mejorar en el uso de esta herramienta, podríamos mencionar el hecho de que se necesite conexión a internet y que los estudiantes dispongan de un smartphone, tablet u ordenador portátil para conectarse; y que en ocasiones la aplicación da errores.

Como líneas futuras de trabajo proponemos el análisis de las calificaciones obtenidas en la asignatura comparando cursos académicos donde no se empleó esta herramienta con este curso académico, puesto que ha sido el primero en el cual se ha utilizado. De esta forma podríamos observar si hay mejora o no de los resultados académicos de los estudiantes. Igualmente, otra línea de trabajo podría ser analizar si existe o no relación positiva entre los resultados de los Kahoot!s y la calificación en la asignatura. 


\section{Referencias}

ALCOVER, R.M., CALDUCH LOSA, A. y VIDAL PUIG, S. (2018). "Nos divertimos y aprendemos con Kahoot! en las clases de estadística” en IN-RED 2018. Congreso Nacional de Innovación Educativa $y$ de Docencia en Red. Valencia: Universitat Politècnica de Valencia. p.165-175.

ADAMS, S., CUMMINS, M., DAVIS, A., FREEMAN, A., HALL, C., y ANANTHANARAYANAN, V. (2017). NMC Horizon Report: 2017 Higher Education Edition. Austin, Texas: The New Media Consortium. <http://www.nmc.org/publication/nmc-horizon-report-2017-higher-education-edition/> [Consulta: 16 de enero de 2019]

BARNES, R. (2017). "Kahoot! in the Classroom: Student Engagement Technique” en Nurse Educator, vol.42, isuee 6 , p. 280.

BRAZUELO GRUND, F., y GALLEGO GIL, D.J. (2011). "Mobile Learning. Los dispositivos móviles como recurso educativo" en RIED: Revista Iberoamericana de Educación a Distancia. n. 14, vol. 2, p. 212-214.

CALDERÓN, C. y GONZÁLEZ, A. (2009). "El papel del profesor universitario español en el EEES. Retos pendientes" en II International Conference: X Seminario Iberoamericano Motiva La Universidad y el Emprendimiento, Valencia, 27-30 octubre.

CHAIYO, Y. y NOKHAM. R. (2017). "The Effect of Kahoot, Quizizz and Google forms on the Student's Perception in the Classrooms Response System" en 2017 Inernational Conference on Digital Arts, Media and Technology (ICDAMT)-Digital Economy for Sustainable Growth. Chiang Mai. p.178182.

DEL CERRO GÓMEZ, G. (2015). “Aprender jugando, resolviendo: diseñando experiencias positivas de aprendizaje" en XII Jornadas Internacionales de Innovación Universitaria (20-21 Julio 2015. Villaviciosa de Odón). Madrid: Universidad Europea de Madrid. p. 237-244.

FUERTES, A., GARCÍA, M., CASTAÑO, M.A., LÓPEZ, E., ZACARES, M., COBOS, M., FERRIS, R. y GRIMALD, F. (2016). "Uso de herramientas de respuesta de audiencia en la docencia presencial universitaria. Un primer contacto" en XXII Jenui (6-8 Julio 2016. Almería). Editorial Universidad de Almería. p. 261-268.

GARCÍA GARCÍA, D., CARBONELL VERDU, A., MONTAÑES, N., QUILES, L. y FOMBUENA, V. (2017). "Incorporación de la aplicación Kahoot! para la evaluación de las prácticas de la asignatura de "Ciencias de Materiales". Fernández y Botti (coord.) en IN-RED 2017. Congreso Nacional de Innovación Educativa y de Docencia en Red. Valencia: Universitat Politécnica de Valencia. p.12.091217. < http://hdl.handle.net/10251/86908> [Consulta: 8 de febrero de 2019].

GÓMEZ TORRES, M.J., SÁEZ ESPINOSA, P., ROBLES GÓMEZ, L., HUERTA RETAMAL, N., ROMERO, A., VELASCO, I., y TORRIJO BOIX, ST. (2018). "Kahoot!! como instrumento de refuerzo en Biología del Desarrollo" en Rosabel Roig-Vila (Ed.). El compromiso académico y social a través de la investigación e innovación educativas en la Enseñanza Superior. Barcelona:Octaedro, SL. p.10851094.

GRINIAS, J.P. (2017). "Making a game out of it: using we-based competitive quizzes for cuantitative analysis content review", Journal of Chemical Education, nº. 94, vol. 9, p. 1363-1366.

IWAMOTO, D.H., HARGIS, J., TAITANO, E.J. y VUONG, K. (2017). "Analizing the efficacy of testing effect using Kahoot Tм oon student performance", Turkish Online Journal of Distance Education, n ${ }^{\circ} 18$, vol. 2, p.80-93. 
KAPP, K.M. (2012). The gamification of Learning and Instruction: game based Methods and Strategies for Training and Education. New York: Pfeiffer-Wiley.

LLERENA, E.G. y RODRIGUEZ, C.P (2017). "Kahoot! A Digital Tool for Learning Vocabulary in a Language Classroom" en Revista Publicando, vol. 4, isuee 12, p. 441-449.

MARÍN SUELVES, D., VIDAL ESTEVE, M.I., PEIRATS CHACÓN, J. y LÓPEZ MARI, M. (2018). "Gamificación en la evaluación del aprendizaje: valoración del uso de Kahoot!!" en Redine (Ed.). Innovative Strategies for Higher Education in Spain. Eindhoven, NL: Adaya Press. p. 8-17.

MONEDERO, C.R., y CASTRO, A. (2018). "Un proyecto de aplicación de la clase invertida en la Ciencias de la Comunicación" en Redine (Ed.). Innovative Strategies For Higher Education in Spain, Eindhoven, NL: Adaya Press. p.69-79.

MU, H.R. y PAPARAS, D. (2015). "Incorporating the advantages of clickers and mobile devices to teach economics to non-economist", Congent Economics\&Finance, n' ${ }^{\circ}$. 3, vol.1, p. 1-10.

PEÑA, B., ZABALZA, I., USÓN, S., LLERA, E.M., MARTÍNEZ, A., ROMEO, L.M. /2017). "Experiencia piloto de aula invertida para mejorar el proceso de enseñanza-aprendizaje en la asignatura de Termodinámica Técnica" en Fernández y Botti (coord..): IN-RED 2017. Congreso Nacional de Innovación Educativa y de Docencia en Red. Valencia: Universitat Politécnica de Valencia. p. 583596. $<$ http://hdl.handle.net/10251/86908> [Consulta: 8 de febrero de 2019].

RODRIGUEZ FERNÁNDEZ, L. (2017). "Smartphone y aprendizaje: el uso de Kahoot! en el aula universitaria" en Revista Mediterránea de Comunicación. Alicante: Universidad de Alicante. vol.8, nº1, p.181-190.

SAINZ DE ABAJO, B., DE LA TORRE DÍEZ, I., LÓPEZ CORONADO, M., COUTO A.C. y DE CASTRO LOZANO, C. (2018). "Gamificación en la evaluación dinámica de contenidos de la materia básica del grado en Criminología" en Vega y Vendrell (ed): IV Congreso Nacional de Innovación Educativa y de Docencia en Red (In-Red, 2018). Valencia: Universitat Politècnica de Valencia. p.804813.

SEMPERE FERRE, F. (2018). "Kahoot como herramienta de autoevaluación en la universidad" en $I N$ RED 2018. Congreso Nacional de Innovación Educativa y de Docencia en Red. Valencia: Universitat Politècnica de Valencia. p. 250-255.

SAN MIGUEL, T., MEGIAS, J. y SERNA, E. (2017). “Gamificación en la universidad II: aprendemos a divertirnos enseñando. Se divierten aprendiendo" en Fernández y Botti (coord..): IN-RED 2017. Congreso Nacional de Innovación Educativa y de Docencia en Red. Valencia: Universitat Politécnica de Valencia. p. 484-492. <http://hdl.handle.net/10251/86908> [Consulta: 8 de febrero de 2019].

VILLALUSTRE MARTÍNEZ, M. L. y MORAL PÉREZ, P. E. (2015). “Gamificación: Estrategia para optimizar el proceso de aprendizaje y la adquisición de competencias en contextos universitarios" en Digital Education. Barcelona: Revistes Cientifiques de la Universitat de Barcelona. n²7, p.13-31. $<$ http://greav.ub.edu/der> [Consulta: 8 de febrero de 2019].

WANG INGE, A, y LIEBEROTH, A. (2016). "The effect of points and audio on concentration, engagement, enjoyment, learning, motivation, and classroom dynamics using Kahoot!" en: Proceeding of the $10^{\text {th }}$ European Conference on Games-Based Learning. Scotland: At.Paisley. p.738-746. 